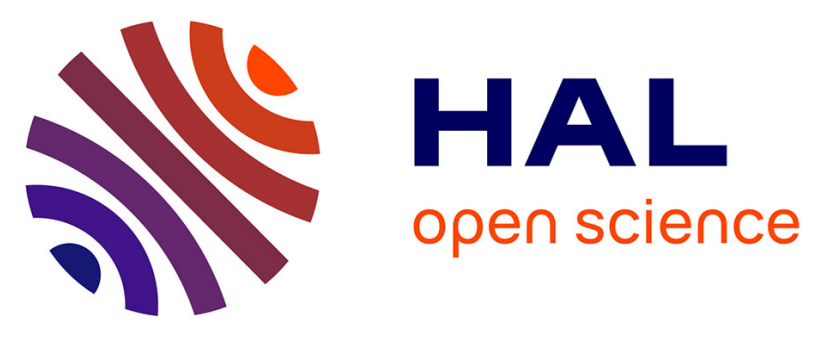

\title{
Production of a novel mixture of mycosubtilins by mutants of Bacillus subtilis
}

Max Béchet, Joany Castéra-Guy, Jean-Sebastien Guez, Nour-Eddine Chihib, Françoise Coucheney, François Coutte, Patrick Fickers, Valérie Leclère, Bernard Wathelet, Philippe Jacques

\section{To cite this version:}

Max Béchet, Joany Castéra-Guy, Jean-Sebastien Guez, Nour-Eddine Chihib, Françoise Coucheney, et al.. Production of a novel mixture of mycosubtilins by mutants of Bacillus subtilis. Bioresource Technology, 2013, 145, pp.264-270. 10.1016/j.biortech.2013.03.123 . hal-02938868

\section{HAL Id: hal-02938868 https://hal.science/hal-02938868}

Submitted on 22 Sep 2020

HAL is a multi-disciplinary open access archive for the deposit and dissemination of scientific research documents, whether they are published or not. The documents may come from teaching and research institutions in France or abroad, or from public or private research centers.
L'archive ouverte pluridisciplinaire HAL, est destinée au dépôt et à la diffusion de documents scientifiques de niveau recherche, publiés ou non, émanant des établissements d'enseignement et de recherche français ou étrangers, des laboratoires publics ou privés. 


\section{Production of a novel mixture of mycosubtilins by mutants of Bacillus} subtilis

Max Béchet ${ }^{\text {a,1 }}$, Joany Castéra-Guy ${ }^{\text {a,1 }}$, Jean-Sébastien Guez ${ }^{\text {a,b }}$, Nour-Eddine Chihib ${ }^{\text {a }}$ Françoise Coucheney ${ }^{a}$, François Coutte $^{a}$, Patrick Fickers ${ }^{c}$, Valérie Leclère ${ }^{a}$, Bernard Wathelet $^{\mathrm{d}}$ and Philippe Jacques ${ }^{\mathrm{a}, *}$

a Laboratoire des Procédés Biologiques, Génie Enzymatique et Microbien-ProBioGEM, UPRES-EA 1026, Polytech'Lille/IUT A, Université Lille Nord de France-Sciences et Technologies, F-59655 Villeneuve d'Ascq Cedex, France

b Axe GePEB, Institut Pascal, UMR 6602, Université Blaise Pascal, CNRS, Clermont Université, BP 10448, F-63000 Clermont-Ferrand, France

${ }^{\mathbf{c}}$ Unité de Biotechnologies et Bioprocédés, Université Libre de Bruxelles, Av. F.-D. Roosevelt 50, C.P. 165/61, B-1050 Bruxelles, Belgium

${ }^{\text {d }}$ Unité de Chimie Biologique Industrielle, Gembloux Agro-BioTech, Université de Liège, B5030 Gembloux, Belgium

* Corresponding author. Address: Laboratoire des Procédés Biologiques, Génie Enzymatique et Microbien, UPRES-EA 1026, Polytech'Lille, Université Lille Nord de France, Lille1, Av. Paul Langevin, F-59655 Villeneuve d'Ascq Cedex, France. Tel.: + 33 328767440; fax: + 33 328767401.

E-mail address: Philippe.Jacques@polytech-lille.fr

${ }^{1}$ Equal contribution as first authors 


\section{ABSTRACT}

Using promoter exchange and gene knock-out strategies, two mutant strains, the so-called BBG116 and BBG125, were constructed from Bacillus subtilis wild-type strain ATCC 6633, a surfactin and mycosubtilin producer. Compared to the parental strain, both mutants overproduced constitutively mycosubtilin, while BBG125 had lost the ability to synthesize surfactin. Surprisingly, BBG125 was found to produce about 2-fold less mycosubtilin than BBG116 despite an expected higher availability of the cytoplasmic precursors and cofactors pool for biosynthesis. Further physiological characterization of BBG125 also highlighted: (i) a strong influence of temperature on mycosubtilin biosynthesis in BBG125 with a maximal productivity observed at $22^{\circ} \mathrm{C}$, compared to 15 and $30^{\circ} \mathrm{C}$; (ii) substantial changes in fatty acid profiles and thereby in mycosubtilin isoforms, compared to the wild-type strain; and (iii) the presence of five novel mycosubtilin isoforms. The antifungal activities of the new mix were higher than or equal to those of purified isoforms.

Key-words: Bacillus subtilis, mycosubtilin, novel isoforms, antifungal activity, spreading

\section{Introduction}

Mycosubtilin is a potent antifungal lipopeptide of the iturin family synthesized by a limited number of Bacillus subtilis (Besson et al., 1978) and Bacillus spp. strains.

Mycosubtilin is composed of a seven amino acid cyclic peptide linked by a lactone ring to a $\beta$-amino fatty acid of variable chain length (i.e., C15 to C17) and isoforms ( $n$-, iso- and anteiso-) (Jacques, 2011). Antifungal activity of mycosubtilin has been shown to be related to 
both the length of the fatty acid component and its isomery (Hbid, 1996). Biosynthesis of mycosubtilin is mediated by a modular multienzymatic complex and the so-called "nonribosomal peptide synthetases" mechanism (NRPS). This multienzymatic complex is encoded by the hybrid PKS/NRPS myc operon of size about $38 \mathrm{~kb}$, which contains four genes (Duitman et al., 1999). Beside this, most of B. subtilis strains coproduce other NRPS compounds such as surfactin, a powerful lipopeptide biosurfactant involved in colonization of surfaces (Leclère et al., 2006) and biofilm formation (Chollet-Imbert et al., 2009), other iturins (iturin A and bacillomycins) or lipopeptides from the fengycin and plipastatin family. Despite the fact that multi-lipopeptide producers, notably in Bacillus amyloliquefaciens, have been isolated (Jacques et al., 1999; Hofemeister et al., 2004; Koumoutsi et al., 2004), the occurrence of strains naturally producing only mycosubtilin has not been reported so far. Interest in Bacillus non-ribosomal lipopeptides both in biocontrol in agricultural area against numerous phytopathogens (e.g., induced systemic resistance in plants) and in potential use as biopesticides, has strongly increased in the recent years (Ongena and Jacques, 2008).

Surfactin synthesis has for a long time been demonstrated to be closely linked to the competence state and sporulation of the cells, and to be subjected to a complex regulatory network involving two groups of pheromones: $\mathrm{ComX}$, on one side, and $\mathrm{PhrC}, \mathrm{PhrF}, \mathrm{PhrG}$ and PhrH on the other side (Hamoen et al., 2003; Hayashi et al., 2006, Jacques, 2011). One of these pheromones, PhrC, would be also involved in the regulation of mycosubtilin biosynthesis. In addition, the strength of the promoters PsrfA and Pmyc, which direct expression of the corresponding operons srfA and $m y c$, is very low (about 200-fold less) in strain ATCC 6633, when compared to the expression of the native PsrfA within the wellknown laboratory strain B. subtilis 168 (Duitman et al., 2007).

In order to demonstrate the role played by mycosubtilin in the biocontrol activity of plant diseases, we previously reported the construction of a B. subtilis ATCC 6633 derivative 
overproducing mycosubtilin, resulting from replacement of the native Pmyc promoter by a constitutive one linked to an antibiotic resistance marker, i.e., the PrepU-neo cassette. This modified strain named BBG100 produced up to 15 -fold more mycosubtilin than the parental strain did, and showed a strongly increased antifungal activity in vivo in the vicinity of tomato roots (Leclère et al., 2005), favoured by the early constitutive and overproducing status of mycosubtilin production. Moreover, a significant improvement of its behaviour for spreading on various solid media was also observed (Leclère et al., 2006).

However, the common use of either precursors such as fatty acids (Kluge et al., 1988) or cofactors such as phosphopantetheine (Vollenbroich et al., 1994) in the syntheses of both mycosubtilin and surfactin by strain ATCC 6633 may limit the mycosubtilin ratio. In addition, it was recently shown that coproduction of mycosubtilin and surfactin led to the formation of mixed micelles which render their separation quite difficult (Jauregi et al., in press). Thus, as a further step to increase mycosubtilin yield and facilitate its purification, the surfactin ( $s r f$ ) operon was inactivated by disrupting its first $s r f A$ gene in a novel constitutive mycosubtilin overproducer. The present paper describes the isolation of such a mutant and the impact of surfactin production suppression on (i) the biosynthesis level and isoforms repartition of mycosubtilin; (ii) the detection of new forms of mycosubtilin; and (iii) the biological activities.

\section{Materials and methods}

\subsection{Bacterial strains, plasmids, and growth conditions}

The microorganisms and plasmids used in this study are listed in Table 1. B. subtilis and E. coli strains were cultivated at $37^{\circ} \mathrm{C}$ in Luria-Bertani medium (LB) supplemented, when 
required, with various antibiotics: ampicillin sodium salt (Ap; Sigma, St. Louis, MO, USA; 50 $\mu \mathrm{g} / \mathrm{mL}$ ), chloramphenicol (Cm; Sigma; $10 \mu \mathrm{g} / \mathrm{mL})$, neomycin trisulfate salt hydrate (Nm; Sigma; $20 \mu \mathrm{g} / \mathrm{mL}$ ), spectinomycin, di-HCl (Spc; Sigma, $50 \mu \mathrm{g} / \mathrm{mL}$ ) and tetracycline-HCl (Tc; Sigma; $10 \mu \mathrm{g} / \mathrm{mL}$ ). B. subtilis strains were also grown either in E medium (Clark et al., 1981) or in $100 \mathrm{mM}$ MOPS-buffered modified Landy medium (Guez et al., 2008) containing $2.3 \mathrm{~g} / \mathrm{L}$ $\left(\mathrm{NH}_{4}\right)_{2} \mathrm{SO}_{4}+2 \mathrm{~g} / \mathrm{L}$ glutamic acid instead of $5 \mathrm{~g} / \mathrm{L}$, at 15,22 or $30^{\circ} \mathrm{C}$ as stipulated in the text.

Soft B medium was used to assess the invasive behaviour of the strains (Coutte et al., 2010a). The yeast strains were cultured at $28^{\circ} \mathrm{C}$ in medium 863 (glucose $10 \mathrm{~g} / \mathrm{L}$, peptone 10 $\mathrm{g} / \mathrm{L}$, yeast extract $10 \mathrm{~g} / \mathrm{L} ; \mathrm{pH} 5.8$ ), whereas the fungal strains were propagated at $30^{\circ} \mathrm{C}$ on non-acidified potato-dextrose-agar, pH 6.0 (PDA; Solabia-Biokar Diagnostics, Beauvais, France), to carry out Bacillus/fungi confrontations.

For lipopeptide production in defined medium, culture flasks were inoculated at an initial biomass equal to an OD 0.25 at $600 \mathrm{~nm}$ (i.e., $0.08 \mathrm{~g}$ D.W/L) from an overnight preculture in E medium. Cultures were performed in triplicate at 15,22 and $30^{\circ} \mathrm{C}$ under rotary shaking $(160 \mathrm{rpm})$.

\subsection{Genetic and molecular biology experiments}

\subsubsection{General genetic techniques}

Standard molecular biology procedures, i.e., total genomic DNA extraction, plasmid extraction, PCR, cloning of PCR amplicon and restriction endonuclease digestions were carried out as described previously (Leclère et al., 2005; Coutte et al., 2010a). B. subtilis strains RFB102 and BBG116 were transformed by either electroporation (Gene Pulser apparatus from Bio-Rad, Hercules, CA, USA) according to the method of Dennis and Sokol (1995) or natural competence (Dubnau, 1982). 


\subsubsection{Strain construction}

The construction of constitutive mycosubtilin-overproducing mutants by double allelic exchange and further mycosubtilin monoproducers needed several steps. First, RFB102 was obtained by transformation of ATCC 6633 with polymers (concatenates) of plasmid ED511, containing $\mathrm{P}_{\text {spac }}$-comK-spc cassette, followed by selection for amylase-negative spectinomycin-resistant clones. These concatenates were obtained with illustra TempliPhi 100 Amplification Kit (GE Healthcare Bio-Sciences Corp., Piscataway, NJ, USA). Then, to construct a constitutive mycosubtilin overproducer mutant, the $2.6 \mathrm{~kb} \varepsilon p b p$-PrepU-neo-हfenF cassette was excised from plasmid pBG106 (Leclère et al., 2005) by a double SacI-SphI digestion and purified. In parallel, a $451 \mathrm{bp}$ fragment carrying the $\gamma$ origin of replication sequence (oriK) from plasmid R6K (unable to function in Bacillus spp.), contained in pTnMod-RKm', was excised from this plasposon using a double NspI-SacI digestion and purified. These two components were ligated and the product was used to transform E. coli $\mathrm{CC} 118(\lambda$ pir $)$ with selection for neomycin resistance, giving rise to plasmid pBG201.

Transformation of $B$. subtilis RFB102 with pBG201 linearized by AatII led to the isolation of several $\mathrm{Nm}^{\mathrm{R}} \mathrm{Spc}^{\mathrm{R}}$ colonies. Correct recombination was verified by analytical PCR using the primers PBP-FO2 and FENF-REV2 (Table 2). Detection of a $\sim 3.6 \mathrm{~kb}$ PCR fragment instead of the $\sim 2.3 \mathrm{~kb}$ one found with the parent strain confirmed the promoter exchange. The resulting strain was denominated BBG116.

For the construction of $\operatorname{srfA}$ disruption cassette, a $2.2 \mathrm{~kb} \varepsilon s r f A A$ fragment was amplified by PCR from B. subtilis 168 genomic DNA using the primers SRF-FO and SRFREV (Table 2) and subsequently cloned into pGEM-T Easy vector to yield plasmid pBG208. The $\varepsilon s r f A A$ fragment was then rescued by EcoRI digestion and subcloned into EcoRI-digested pUC19, to give rise to plasmid pBG210 (4.9 kb). 
Beside this, a tet gene was amplified by PCR using primers TetP1 and TetT1 and vector pBC16 as a template, before being cloned into pGEM-T Easy to give rise to plasmid pBG209. The tet gene from this latter plasmid was rescued by EcoRI digestion and subcloned in pBG210 at the $M f e I$ restriction site located within the $\varepsilon s r f A A$ fragment. In a second step, a cat gene was obtained by PCR using the primers pC194cmfwd and pC194cmrev (containing an artificial BstEII site) and pC194 as a template before being cloned into pGEM-T Easy to give rise to pBG143. Digestion of this latter plasmid by BstEII generated a cat gene-bearing fragment which was inserted at the BstEII site situated at the end of the tet gene in pBG212 (without removing the tetracycline-resistance phenotype), giving rise to plasmid pBG144. The mutant BBG116 was then transformed with AatII-linearized pBG144, followed by selection either on LB Cm10 or on LB Tc10 plates, and checking for presence of the associated marker. The resulting strain unable to produce surfactin was called BBG125.

\subsection{Cellular fatty acids analysis}

The cells (from $100 \mathrm{~mL}$ cultures) were harvested by centrifugation at $10,000 \mathrm{~g}$ for 10 min. The pellets were washed twice with sterile distilled water and transferred into extraction screw-cap tubes. Bacteria were subsequently submitted to saponification and methylation and their fatty acid methyl esters were extracted as described by Miller and Berger (1985).

Methanol, hexane and ter-butylmethylether were of analytical grade (Merck, Darmstadt, Germany).

Analysis of fatty acid methyl esters was performed using a GC-2014 gas chromatograph (Shimadzu, Kyoto, Japan) as previously described (Chihib et al., 2003). At least two samples were withdrawn for each challenge condition and each experiment was done in triplicate. 


\subsection{Lipopeptide purification and analyses}

\subsubsection{Purification for HPLC analysis}

Lipopeptides were purified from the culture supernatant by solid phase extraction using a 500 mg C18 Maxi-Clean cartridge (Grace Davison-Alltech, Deerfield, IL, USA) according to Guez et al. (2007). Briefly, after extraction of lipopeptide from $1 \mathrm{~mL}$ supernatant sample, the cartridge was washed with $8 \mathrm{~mL}$ of water. The lipopeptides were then eluted with $8 \mathrm{~mL}$ of $100 \%$ methanol (HPLC grade, Acros Organics, Geel, Belgium) and the extract was brought to dryness before dissolution in $200 \mu \mathrm{L} 100 \%$ methanol at $4^{\circ} \mathrm{C}$.

Lipopeptides were quantified by high-performance liquid chromatography (Online Degaser, 717 Autosampler, 660S Controller, 626 Pump, 996 PhotoDiodeArray, Waters Corp., Milford, MA, USA) using a C18 column $(5 \mu \mathrm{m}, 250$ x $4.6 \mathrm{~mm}, 218 \mathrm{TP}$, VYDAC, Grace Davison-Alltech). Lipopeptides from a $10 \mu \mathrm{L}$ sample were eluted at a flow rate of $0.6 \mathrm{~mL} / \mathrm{min}$ using an acetonitrile/water/trifluoroacetic acid (TFA) mixture (40:60:0.5; V/V/V) for mycosubtilins, and another acetonitrile/water/ TFA mixture (80:20:0.5; V/V/V) for surfactins. Purified iturin A (Sigma-Aldrich, St Louis, MO, USA), mycosubtilins and surfactins (purified at ProBioGEM as previously described in Coutte et al., 2010b) were used as standards. The retention time and the second derivative of the absorption spectrum between 200 and $400 \mathrm{~nm}$ (Diode Array PDA 996, Waters Corp.) were used to identify the eluted molecules (Millenium Software, Waters Corp.).

\subsubsection{Preparation for MALDI-TOF-MS analysis}

A volume of $10 \mathrm{~mL}$ at $\mathrm{pH} 6.5+/-1$ of the supernatant was purified through a $10 \mathrm{~g} \mathrm{C} 18$ Extract-Clean cartridge (Grace Davison-Alltech), which was then washed with $50 \mathrm{~mL}$ of 
water. The lipopeptides were eluted with $10 \mathrm{~mL}$ of $100 \%$ methanol. The extract was brought to dryness before dissolution in $200 \mu \mathrm{L} 100 \%$ methanol at $4^{\circ} \mathrm{C}$.

A sample volume of $100 \mu \mathrm{L}$ was then injected at $4.5 \mathrm{~mL} / \mathrm{min}$ and analysed by highperformance liquid chromatography as described above, using a C18 column $(5 \mu \mathrm{m}, 300 \times 10$ mm, ACE). A gradient-based method was used for lipopeptide separation. Solvent A (acetonitrile/TFA 99.9:0.1; V/V) was mixed with solvent B (water/TFA 99.9:0.1; V/V). After 4 min in isocratic mode ( $35 \% \mathrm{ACN}$ ), solvent $\mathrm{A} / \mathrm{B}$ ratio increased linearly from $35 / 65$ to $50 / 50$ in $53 \mathrm{~min}$, followed by $50 / 50$ to 100 in $3 \mathrm{~min}$, and then $5 \mathrm{~min}$ in isocratic mode (35\% $\mathrm{ACN}$ ).

\subsubsection{MALDI-TOF mass spectrometry analyses}

MALDI-TOF mass spectrometry analyses were done on three different types of samples: (i) raw culture supernatants, (ii) samples purified through ODS cartridges (Grace Davison-Alltech) or (iii) samples purified through ODS cartridges, followed by semipreparative HPLC. A saturated solution of $\alpha$-cyano-4-hydroxy-cinnamic acid (CHCA) was prepared in a 1:2(V/V) solution of $\mathrm{CH}_{3} \mathrm{CN}$ and $\mathrm{H}_{2} \mathrm{O}$ containing $0.1 \%$ TFA. The previous solutions were diluted 10-fold with CHCA-saturated solution. Then, $0.5 \mu \mathrm{L}$ of this solution was deposited on the target. Measurement was performed using a MALDI-TOF spectrometer (Bruker UltraFlex TOF; Bruker Daltonics Inc., Billerica, MA, USA) equipped with a pulsed nitrogen laser $(\lambda=337 \mathrm{~nm})$. The ions were extracted from the ionization source with an acceleration voltage of $20 \mathrm{kV}$. Samples were measured in the reflectron mode, positive mode. The instrument was externally calibrated with a peptide mix ranging from $1046.54 \mathrm{~m} / \mathrm{z}$ to $3147.47 \mathrm{~m} / \mathrm{z}$ (Bruker part. 206195). The spectrum was obtained with $5 \times 30$ shots on the sample.

\subsubsection{Tandem mass spectrometry MS-MS analyses}


To determine precisely the different forms of mycosubtilin produced by BBG125 strain, purified samples were analysed by tandem mass spectrometry (MS-MS) with electrospray and ion trap. Measurements were made by direct infusion of the open peptide after treatment with N-bromosuccinimide (Peypoux et al., 1981). The dry peptide was treated with $20 \mu \mathrm{L}$ of a solution containing an equal amount of $\mathrm{N}$-bromosuccinimide in $70 \%$ acetic acid for $3 \mathrm{~h}$ at room temperature. The excess of reagent was destroyed by $5 \mu \mathrm{L}$ formic acid. The hydrolysate was evaporated to dryness. Time-dependent MS-MS was made with a Bruker Esquire HCT ESI-mass spectrometer. The conditions of the electrospray were: $180 \mu \mathrm{L} / \mathrm{h}$ flowrate; ultrascan mass range mode; positive ion polarity; ESI source; $300{ }^{\circ} \mathrm{C}$ dry temperature; $15 \mathrm{psi}_{2}$ at nebulizer; $4 \mathrm{~L} / \mathrm{min}$ dry gas; 4,000 V for $\mathrm{HV}$ capillary.

\subsection{Analyses of antimicrobial activities, invasive behaviour, and determination of minimal} inhibitory concentrations

Antimicrobial activities and invasive behaviour were analysed as described previously (Coutte et al., 2010a). In addition, antifungal activity tests against $B$. cinerea were performed by successive half-dilutions of various solutions of mycosubtilin isoforms (iso-C16, $n$-C16, anteiso-C17 and iso-C17) in 96-well microplates containing a rich medium, according to Besson et al. (1979). A mycosubtilin mix (MS comp.) was also tested, composed of (\% w/w): iso-C16 (26\%), Gln3 C17 (1\%), $n$-C16 (2\%), anteiso-C17 (44\%), iso-C17 (23\%) and $\mathrm{C} 18$ $(1 \%)$. Growth was observed after $24 \mathrm{~h}$ at $28^{\circ} \mathrm{C}$, and the minimum inhibitory concentration (MIC) was determined.

\section{Results and discussion}




\subsection{Construction of constitutive mycosubtilin-overproducing mutants by double allelic}

exchange and generation of a mycosubtilin monoproducer

B. subtilis ATCC 6633 is known for its very low transformation frequency by natural competence, probably due to the lack of a functional $\operatorname{comK}$ gene. To bypass this technical drawback, the two mutants obtained in this study were based on the RFB102 strain, an inducible competent derivative of ATCC 6633, generated by integration of a $\mathrm{P}_{\text {spac }}$-comK gene at the $a m y E$ locus. Thus, in the presence of IPTG, RFB102 harbours a competent phenotype and is less refractory to transformation.

The previously constructed mycosubtilin constitutive overproducer mutant BBG100 (Leclère et al., 2005) was observed to be slightly unstable after repeated cultivations, and to have been generated by a single crossing-over event (Campbell-type recombination; results not shown). Therefore, construction of a novel mutant was undertaken. Natural competencemediated transformation of B. subtilis RFB102 with AatII-linearized pBG201 led to isolation of mutant BBG116 (Srf+ Myc++). PCR controls confirmed the occurrence of a double crossing-over event in this $\mathrm{Nm}^{\mathrm{R}} \mathrm{Spc}^{\mathrm{R}}$ derivative (data not shown). BBG116 was found to produce up to eight to ten-fold more mycosubtilin than the parent strain RFB102, which are results similar to those obtained with strain BBG100.

After further transformation of BBG116 by natural competence with AatII-linearized pBG144, the mycosubtilin monoproducer BBG125, which proved to be stable upon repeated cultivations, was isolated. Insertion of the cat-tet cassette within its $\operatorname{srfAA}$ gene was checked by PCR using the primers SRFAA5-FWD and SRFAA5-REV (Table 2): a $\sim 4.7 \mathrm{~kb}$ amplicon was obtained instead of the $\sim 2.3 \mathrm{~kb}$ one found with the parent strains (data not shown). Analysis of mycosubtilin production, carried out after 4 days of incubation at $30^{\circ} \mathrm{C}$ in Landy modified medium ( $200 \mathrm{~mL}$ in a $1 \mathrm{~L}$ flask), revealed that BBG125 synthesized about 2-fold 
less mycosubtilin $(58.9 \pm 6.2 \mathrm{mg} / \mathrm{L})$ than its parent BBG116 $(134.1 \pm 11.1 \mathrm{mg} / \mathrm{L}$; together with $69.3 \pm 9.1 \mathrm{mg} / \mathrm{L}$ of surfactin). Despite an expected higher availability of precursors/cofactors for mycosubtilin production, these results suggested that a functional surfactin biosynthesis should act positively on mycosubtilin production. Moreover, the fact that the knock-out of $s r f A A$ gene was located upstream from the second $s r f A B$ gene, within which the $\mathrm{P}_{s r f A}$-driven regulatory comS gene is nested (D'Souza et al., 1994), might induce a negative impact on mycosubtilin production.

\subsection{Influence of temperature on growth and mycosubtilin production of strain BBG125}

\subsubsection{Effect of temperature changes on the growth of $\mathrm{B}$. subtilis ATCC 6633 and its derivative}

\section{$B B G 125$}

In a previous work (Fickers et al., 2008), the influence of temperature on mycosubtilin production by $B$. subtilis ATCC 6633 was demonstrated. In order to characterize further this influence, the constitutive monoproducer BBG125 was cultivated at three different temperatures $\left(15,22\right.$ and $\left.30^{\circ} \mathrm{C}\right)$ under rotary shaking conditions in Erlenmeyer flasks. The growth curve was first compared to that of $B$. subtilis ATCC 6633 under these different conditions. In the experiment performed at $22^{\circ} \mathrm{C}$, a diauxic growth was observed for both strains. This diauxic growth results from a consumption of glucose (during the four to nine first hours of culture) accompanied by a strong production of acidic compounds which were re-consumed in a second phase (up to $24 \mathrm{~h}$ of culture, data not shown). BBG125 was found to grow slower than ATCC 6633 during the early stage of exponential phase $\left(0.17\right.$ and $0.31 \mathrm{~h}^{-1}$, respectively) until reaching a similar population at the stationary phase. The same slowdown of BBG125 growth compared to ATCC 6633 was observed at every temperature as shown in Table 3. Additionally, the growth of both strains at $15^{\circ} \mathrm{C}$ was found to be much slower than at 
higher temperatures, revealed by a dilatory exponential phase.

\subsubsection{Effect of temperature on mycosubtilin production in B. subtilis ATCC 6633 and its} mutant BBG125

Mycosubtilin production was monitored during the growth of both strains by measuring its concentration in the culture supernatant at various time periods. Production of mycosubtilin was found maximal during the stationary phase. Moreover, mycosubtilin specific productivity was more important for BBG125 at $22^{\circ} \mathrm{C}$ than for ATCC 6633, up to $132.25 \pm 3.83 \mathrm{mg} / \mathrm{L}$ mycosubtilin being obtained (Table 3 ). This behaviour was observed from the beginning of the exponential growth phase $(27.7 \mathrm{mg} / \mathrm{L}$ for BBG125 after $15.3 \mathrm{~h}$ of cultivation instead of $7.1 \mathrm{mg} / \mathrm{L}$ for ATCC 6633 after $21.6 \mathrm{~h}$ of cultivation), demonstrating immediate mycosubtilin production due to the presence of the constitutive $\mathrm{P}_{\text {repU }}$ promoter. This increased production could be observed throughout the culture, reaching a maximal productivity up to 4 times higher than for ATCC 6633. Mycosubtilin maximal concentration in BBG125 increased twice when the growth temperature decreased from $30^{\circ} \mathrm{C}$ to $22^{\circ} \mathrm{C}$

(Table 3). Under the same conditions, the maximal specific productivity increased 4 times for BBG125. Moreover, when the cells were grown at $15^{\circ} \mathrm{C}$, an equivalent specific productivity as at $22^{\circ} \mathrm{C}$ was observed.

\subsubsection{Effect of growth temperatures on fatty acid and mycosubtilin patterns}

The changes of growth temperature affected the relative amount of homologues of fatty acids in both the wild-type and the mutant strains. When the growth temperature decreased from $30^{\circ} \mathrm{C}$ to $15^{\circ} \mathrm{C}$, the anteiso to iso fatty acids ratio increased in response to a decrease in the cellular concentration of the iso fatty acids (C14, C15, C16 and C17) homologues and an increase in anteiso (C15 and C17) homologues. Interestingly, this 
behaviour was comparatively more marked for BBG125 (ratios from 3.3 to 24.5 ) than for ATCC 6633 (ratios from 2.3 to 4.1 ). In fact, at $30^{\circ} \mathrm{C}$, the ratios were similar for both strains but at lower temperatures, the difference between the anteiso to iso fatty acids ratios of both strains increased. Thus, at $22^{\circ} \mathrm{C}$, the ratio was 2.6 times higher for the mutant $\mathrm{BBG} 125$ and, at $15^{\circ} \mathrm{C}$, the ratio was 5.4 times higher. It was established by Klein et al. (1999), that the growth temperature induced changes in cellular fatty acids profile of $B$. subtilis towards even iso, odd iso and anteiso chain. In fact, in response to a cold stress, $B$. subtilis acclimates by changing the lipid composition of its cell membrane, avoiding a decrease of membrane fluidity. This adjustment is mainly reflected by increasing the proportion of anteiso branched fatty acids.

Analysis of culture supernatants by RP-HPLC revealed that the mycosubtilin production of BBG125 showed an increase of the anteiso to iso mycosubtilin ratio in regard to the ATCC 6633 production at each tested temperature. Moreover, at low temperatures $\left(22^{\circ} \mathrm{C}\right.$ and $\left.15^{\circ} \mathrm{C}\right)$, the difference between the anteiso to iso mycosubtilin ratio for the two strains was more significant, nearly four times higher for BBG125 at $15^{\circ} \mathrm{C}(10.0$ instead of 2,6 for ATCC 6633). Finally, for both strains, an increase in the anteiso to iso mycosubtilin ratio was observed when the growth temperature decreased, owing to an increase in mycosubtilin anteiso-C17 proportion and a slight decrease in iso proportions. It has been reported that the $B$. subtilis mycosubtilin production pattern shows an increase in the proportion of the $\mathrm{C} 17$ homologues when $B$. subtilis grows at low temperatures (Fickers et al., 2008).

\subsection{Detection and characterization of new forms of mycosubtilin}

The combined HPLC and MALDI-TOF mass spectrometry analyses performed on culture supernatant of BBG125 revealed ten peaks, whose molecular masses are presented in 
Table 4. MS-MS analyses were performed for each peak, the results for peaks 8, 6 and 10 being detailed.

The MS1 spectrum of the cyclic product of peak 8 gave peaks at m/z $1085[\mathrm{M}+\mathrm{H}]^{+}$, $1107[\mathrm{M}+\mathrm{Na}]^{+}$et $1123[\mathrm{M}+\mathrm{K}]^{+}$corresponding well to $\mathrm{C} 17$ mycosubtilin. The spectrum (MS1) of the open structure of peak 8 (with $\mathrm{N}$-bromosuccinimide) was complicated by the two $\mathrm{Br}$ isotopes. Owing to ${ }^{79} \mathrm{Br}$ and ${ }^{81} \mathrm{Br}$ isotopes in about natural equal amount, a peak distribution was observed around $\mathrm{m} / \mathrm{z} 1260$ corresponding to the expected derivative. For example, the peak at $\mathrm{m} / \mathrm{z} 1257.4$ is the $[\mathrm{M}+\mathrm{H}]^{+}$molecule with two ${ }^{79} \mathrm{Br}$, the peak at $\mathrm{m} / \mathrm{z} 1259.4$ is the $[\mathrm{M}+\mathrm{H}]^{+}$molecule with one ${ }^{79} \mathrm{Br}$ and one ${ }^{81} \mathrm{Br}$ or $\left.[\mathrm{M}+\mathrm{H}]\right]^{+}$molecule with two ${ }^{13} \mathrm{C}$ and two ${ }^{79} \mathrm{Br}$, etc... The MS2 spectrum of peak 8 open structure is complicated by the presence of fragments with a loss of 1,2 or $3 \mathrm{NH}_{3}$, owing to the presence of Asn and Gln amino acids. Increasing $\mathrm{m} / \mathrm{z}$ obtained with the fragmentation $\mathrm{m} / \mathrm{z} 1260.4$ ion with formula NQPSNxNz (with $\mathrm{x}$ for fatty amino acid and $\mathrm{z}$ for brominated Tyr) is presented in Table 5.

The MS analysis of peak 6 showed that the peptide had an isotopic mass of 1098.6, while the open peptide (with $\mathrm{N}$-bromosuccinimide) had a mass of 1270.4 with two ${ }^{79} \mathrm{Br}$. The $\mathrm{y}$ fragments were identical to anteiso-C17 mycosubtilin while the $\mathrm{b}$ fragments were $14 \mathrm{~m} / \mathrm{z}$ above $\mathrm{b}$ fragments of anteiso-C17 mycosubtilin. This meant that one of the first two amino acids in the open sequence ( $\mathrm{N}$ or $\mathrm{Q}$ from NQPSNxNz) had been modified. The substitution of an Asn by a Gsn is the sole retained reasonable hypothesis.

The MS analysis of peak 10 showed that the peptide had an isotopic mass of 1098.6, while the open peptide (with N-bromosuccinimide) had a mass of 1270.4 with two ${ }^{79} \mathrm{Br}$. The y fragments were $14 \mathrm{~m} / \mathrm{z}$ above the $\mathrm{y}$ fragments of anteiso-C17 mycosubtilin. The $\mathrm{b}_{\mathrm{n}}$ fragments, with $\mathrm{n}$ less than 6 , were identical to anteiso-C17 mycosubtilin. $\mathrm{B}_{\mathrm{n}}$ fragments with $\mathrm{n}$ equal or over 6 , had the same mass plus $14 \mathrm{~m} / \mathrm{z}$. These results showed that the molecule should be mycosubtilin with a C18 and not a C17 fatty acid. 
On the basis of HPLC elution of the peaks, we deduced the existence of 5 new forms of mycosubitilin: (i) four forms with Gln in position 3: iso-C16, $n$-C16, anteiso-C17, iso-C17 and (ii) one form with C18. The correspondence of these forms with the ten HPLC peaks is presented in Table 4. These forms which are produced in low amounts (about $1 \%$ of the total mixture), compared to the main forms already identified, probably result from the ability of some domains of NRPS involved in the biosynthesis of mycosubtilin to recognize different substrates, especially the adenylation domain of module 3 which could be able to activate Asn and Gln residues.

\subsection{Biological activities of the mutants compared to parent strain}

B. subtilis ATCC 6633 and its three derivatives (RFB102, BBG116 and BBG125) were tested for (i) their antagonism versus two fungi (Botrytis cinerea and Fusarium oxysporum) and the yeast Saccharomyces cerevisiae, and (ii) their invasive behaviour on soft B medium. In all cases, mutant BBG116 ( $\mathrm{Srf}+\mathrm{Myc}++)$ proved to be the most efficient strain, owing to the higher amount of mycosubtilin produced by this strain but probably also by a synergistic effect between surfactin and mycosubtilin (Maget-Dana et al., 1992). The derivative BBG125 (Srf- Myc+) was observed to display the second significant antifungal activity, due to mycosubtilin overproduction, compared to strains ATCC 6633 and RFB102 (data not shown). Thanks to surfactin, a strong biosurfactant implied in colonization of surfaces mechanisms (spreading, swarming,...), the wild-type 6633 was the second best invasive strain (Fig. 1). These results are in agreement with the correlation between enhanced spreading (and thereby colonizing behaviour) and surface-active properties displayed by these two lipopeptides (Leclère et al., 2006). 
Against B. cinerea, the minimal inhibitory concentrations of (i) individual purified isoforms of mycosubtilin and (ii) a mix of several isoforms, were also determined. The overall results showed that the isoforms mix gave equal or stronger effects, compared to the individual isoforms ones. Indeed, the anteiso-C17 isoform and the mix (MS comp.) were the most active against the fungus (MIC of $8 \mu \mathrm{M}$ each), followed by the $n-\mathrm{C} 16$ and iso-C17 isoforms (MIC of $16 \mu \mathrm{M}$ each), and the iso-C16 one (MIC of $32 \mu \mathrm{M}$ ). The presence of small amounts (1\% each) of the new isoforms Gln3 $\mathrm{C} 17$ and $\mathrm{C} 18$ in the mix significantly improved its activity, suggesting an even more powerful activity for these variants alone.

\section{Conclusion}

For the first time, the construction of a $B$. subtilis constitutive mycosubtilin monoproducer is reported here. Although mycosubtilin productivity was lowered by the suppression of the capacity of surfactin synthesis, the mutant BBG125 produced a novel mixture of mycosubtilins with five new isoforms. In addition, it displayed substantial changes in both fatty acids profiles and mycosubtilin isoforms, compared to the original strain ATCC 6633. Significant antimicrobial activities were observed against filamentous fungi and a yeast, showing that BBG125 and its surfactin-producing parent (BBG116) were potent candidates as biocontrol agents.

\section{Acknowledgements}

This work received financial support from the Université Lille 1, Sciences et Technologies and ARCIR funds from Nord-Pas-de-Calais and the European Funds of INTERREG IV PhytoBio Project. We thank Corinne Boistel and Laurent Bonneau for their 
precious help in lipopeptide production. David Rudner from the Department of Microbiology and Immunobiology at Harvard Medical School is acknowledged for providing ED511 vector bearing the Pspac-comK construct, and William Everett for his kind English proofreading.

\section{References}

[1] Bernhard, K., Schrempf, H., Goebel, W., 1978. Bacteriocin and antibiotic resistance plasmids in Bacillus cereus and Bacillus subtilis. J. Bacteriol. 133, 897-903.

[2] Besson, F., Peypoux, F., Michel, G., Delcambe, L., 1978. Identification of antibiotics of iturin group in various strains of Bacillus subtilis. J. Antibiot. (Tokyo) 31, 284-288.

[3] Besson, F., Peypoux, F., Michel, G., Delcambe, L., 1979. Antifungal activity upon Saccharomyces cerevisiae of iturin A, mycosubtilin, bacillomycin L and of their derivatives; inhibition of this antifungal activity by lipid antagonists. J. Antibiot. (Tokyo). 1979, 828-833.

[4] Chihib, N.-E., Ribeiro da Silva, M., Delattre, G., Laroche, M., Federighi, M., 2003. Different cellular fatty acid pattern behaviours of two strains of Listeria monocytogenes Scott A and CNL 895807 under different temperature and salinity conditions. FEMS Microbiol. Lett. 218, 155-160.

[5] Chollet-Imbert, M., Gancel, F., Slomianny, C., Jacques, P., 2009. Differentiated pellicle organization and lipopeptide production in standing culture of Bacillus subtilis strains. Arch. Microbiol. 191, 63-71.

[6] Clark, J.B., Munnecke, D.M., Jenneman, G.E., 1981. In situ microbial enhancement of oil 
recovery. Dev. Ind. Microbiol. 22, 695-701.

[7] Coutte, F., Leclère, V., Béchet, M., Guez, J.-S., Lecouturier, D., Chollet-Imbert, M., Dhulster, P. Jacques, P., 2010a. Effect of pps disruption and constitutive expression of $s r f A$ on surfactin productivity, spreading and antagonistic properties of Bacillus subtilis 168 derivatives. J. Appl. Microbiol. 109, 480-491.

[8] Coutte, F., Lecouturier, D., Ait Yahia, S., Leclère, V., Béchet, M., Jacques, P., Dhulster P., 2010b. Production of surfactin and fengycin by Bacillus subtilis in a bubbleless membrane bioreactor. Appl. Microbiol. Biotechnol. 87, 499-507.

[9] Dennis, J.J., Sokol, P.A., 1995. Electrotransformation of Pseudomonas. Methods Mol. Biol. 47, 125-133.

[10] Dennis, J.J., Zylstra, G.J., 1998. Plasposons: modular self-cloning minitransposon derivatives for rapid genetic analysis of gram-negative bacterial genomes. Appl. Environ. Microbiol. 64, 2710-2715.

[11] D'Souza, C., Nakano, M.M., Zuber, P., 1994. Identification of comS, a gene of the srfA operon that regulates the establishment of genetic competence in Bacillus subtilis. Proc. Natl. Acad. Sci. USA 91, 9397-9401. Erratum in: Proc. Natl. Acad. Sci. USA, 1995, 92, 646.

[12] Dubnau, D.A., 1982. Genetic transformation of Bacillus subtilis, in: Dubnau, D.A. (Ed.), The Molecular Biology of the Bacilli, vol I. Bacillus subtilis. Academic Press Inc., New York, pp. 148-178. 
[13] Duitman, E.H., Hamoen, L.W., Rembold, M., Venema, G., Seitz., H, Saenger., W, Bernhard, F., Reinhardt, R., Schmidt, M., Ullrich, C., Stein, T., Leenders, F., Vater, J., 1999. The mycosubtilin synthetase of Bacillus subtilis ATCC6633: a multifunctional hybrid between a peptide synthetase, an amino transferase and a fatty acid synthase. Proc. Natl. Acad. Sci. USA 96, 13294-13299.

[14] Duitman, E.H., Wyczawski, D., Boven, L.G., Venema, G., Kuipers, O.P., Hamoen, L.W., 2007. Novel methods for genetic transformation of natural Bacillus subtilis isolates used to study the regulation of the mycosubtilin and surfactin synthetases. Appl. Environ. Microbiol. 73, 3490-3496.

[15] Ehrlich, S.D., 1977. Replication and expression of plasmids from Staphylococcus aureus in Bacillus subtilis. Proc. Natl. Acad. Sci. USA 74, 1680-1682.

[16] Fickers, P., Leclère, V., Guez, J.-S., Béchet, M., Coucheney, F., Joris, B., Jacques, P., 2008. Temperature dependence of mycosubtilin homologue production in Bacillus subtilis ATCC 6633. Res. Microbiol. 159, 449-457.

[17] Guez, J.-S., Chenikher, S., Cassar, J. P., Jacques, P, 2007. Setting up and modelling of overflowing fed-batch cultures of Bacillus subtilis for the production and continuous removal of lipopeptides. J. Biotechnol. 131, 67-75.

[18] Guez, J.-S., Müller, C.H., Danze, P.M., Büchs, J., Jacques, P., 2008. Respiration activity monitoring system (RAMOS), an efficient tool to study the influence of the oxygen rate on 
the synthesis of lipopeptide by Bacillus subtilis ATCC6633. J. Biotechnol. 134, 121-126.

[19] Hamoen, L.W., Venema, G., Kuipers, O.P., 2003. Controlling competence in Bacillus subtilis: shared use of regulators. Microbiology 149, 9-17.

[20] Hayashi, K., Kensuke, T., Kobayashi, K., Ogasawara, N., Ogura, M., 2006. Bacillus subtilis $\mathrm{RghR}(\mathrm{YvaN})$ represses $\operatorname{rap} G$ and $r a p H$, which encode inhibitors of expression of the srfA operon. Mol. Microbiol. 59, 1714-1729.

[21] Hbid, C., 1996. Contribution à l'étude de la relation entre la structure des lipopeptides de Bacillus subtilis et leurs activités hémolytique et antifongique. $\mathrm{PhD}$ thesis, Liège University, Belgium.

[22] Herrero, M., de Lorenzo, V., Timmis, K.N., 1990. Transposon vectors containing nonantibiotic resistance selection markers for cloning and stable chromosomal insertion of foreign genes in Gram-negative bacteria. J. Bacteriol. 172, 6557-6567.

[23] Hofemeister, J., Conrad, B., Adler, B., Hofemeister, B., Feesche, J., Kucheryava, N., Steinborn, G., Franke, P., Grammel, N., Zwintscher, A., Leenders, F., Hitzeroth, G., Vater, J., 2004. Genetic analysis of the biosynthesis of non-ribosomal peptide- and polyketide-like antibiotics, iron uptake and biofilm formation by Bacillus subtilis A1/3. Mol. Genet. Genomics 272, 363-378. 
[24] Jacques, P., Hbid, C., Destain, J., Razafindralimbo, H., Paquot, M., De Pauw, E., Thonart,

P., 1999. Optimization of biosurfactant lipopeptide production from Bacillus subtilis S499 by Plackett-Burman design. Appl. Biochem. Biotechnol., 77-79, 223-233.

[25] Jacques, P., 2011. Surfactin and other lipopeptides from Bacillus spp. In: Soberon-

Chavez, G. (Ed.), Biosurfactants Microbiology Monographs, Vol. 20, Springer-Verlag, Berlin Heidelberg, pp 57-91.

[26] Klein, W., Weber, M. H., Marahiel, M.A., 1999. Cold shock response of Bacillus subtilis: isoleucine-dependent switch in the fatty acid branching pattern for membrane adaptation to low temperatures. J. Bacteriol. 181, 5341-5349.

[27] Kluge, B., Vater, J., Salnikow, J., Eckart, K., 1988. Studies on the biosynthesis of surfactin, a lipopeptide antibiotic from Bacillus subtilis ATCC 21332. FEBS Lett. 231, 107110.

[28] Koumoutsi, A., Chen, X.-H., Henne, A., Liesegang, H., Hitzeroth, G., Franke, P., Vater, J., Borriss, R., 2004. Structural and functional characterization of gene clusters directing nonribosomal synthesis of bioactive cyclic lipopeptides in Bacillus amyloliquefaciens strain FZB42. J. Bacteriol. 186, 1084-1096.

[29] Leclère, V., Béchet, M., Adam, A., Guez, J.-S., Wathelet, B., Ongena, M., Thonart, P., Gancel, F., Chollet-Imbert, M., Jacques, P., 2005. Mycosubtilin overproduction by Bacillus 
subtilis BBG100 enhances the organism's antagonistic and biocontrol activities. Appl. Environ. Microbiol. 71, 4577-4584.

[30] Leclère, V., Marti, R., Béchet, M., Fickers, P., Jacques, P., 2006. The lipopeptides mycosubtilin and surfactin enhance spreading of Bacillus subtilis strains by their surfaceactive properties. Arch. Microbiol. 186, 475-485.

[31] Maget-Dana, R., Thimon, L., Peypoux, F., Ptak, M., 1992. Surfactin/iturin A interactions may explain the synergistic effect of surfactin on the biological properties of iturin A. Biochimie 1992, 1047-1051.

[32] Miller, L., Berger, T., 1985. Bacteria identification by gas chromatography of whole cell fatty acids. Hewlett-Packard application note, pp. 228-241.

[33] Ongena, M., Jacques, P., 2008. Bacillus lipopeptides: versatile weapons for plant disease biocontrol. Trends Microbiol. 16, 115-125.

[34] Peypoux, F., Besson, F., Michel, G., Delcambe, L., 1981. Structure of bacillomycin D, a new antibiotic of the iturin group. Eur. J. Biochem. 118, 323-327.

[35] Vollenbroich, D., Mehta, N., Zuber, P., Vater, J., Kamp, R.M., 1994. Analysis of surfactin synthetase subunits in srfA mutants of Bacillus subtilis OKB105. J. Bacteriol. 176, 395-400. Erratum in: J. Bacteriol. 176, 2136. 
Table 1.

Strains and plasmids.

Strain or plasmid Description*

Reference or source

E. coli CC118( $\lambda$ pir $)$ Host strain for plasmids carrying an R6K-derived Herrero et al. (1990) replicon; $\Delta$ (ara-leu) araD $\Delta$ lacX74 galE galK

phoA20 thi-1 rpsE rpoB argE(Am) recA1 ( $\lambda$ pir)

Rif $^{R}$

E. coli JM109 General purpose cloning strain; recA1 supE44 Promega Corp., endA1 hsdR17 gyrA96 relAl thi $\Delta($ lac-proAB) F' Madison, WI, USA [traD36 proAB lacI $^{q}$ lacZ $\left.\Delta \mathrm{M} 15\right]$

Bacillus subtilis 168 Laboratory strain; $\operatorname{trpC2} \operatorname{deg} Q^{o} s f p^{0}$; Srf- Pps- $\quad$ Lab stock

B. subtilis ATCC Produces mycosubtilin (Myc+), surfactin ( $\mathrm{Srf}+)$, Duitman et al.

$6633 \quad$ subtilin, subtilosin and rhizocticins

B. subtilis RFB102 ATCC 6633 derivative with a $\mathrm{P}_{\text {spac }}$-comK-spc $\quad$ This work cassette inserted at the amyE locus; Myc+

$\mathrm{Srf}+\mathrm{Amy}^{-} \mathrm{Spc}^{\mathrm{R}}$

B. subtilis BBG116 RFB102 derivative overproducing mycosubtilin; This work $\mathrm{Myc}++\mathrm{Srf}+\mathrm{Amy}^{-} \mathrm{Spc}^{\mathrm{R}} \mathrm{Nm}^{\mathrm{R}} ;\left[\mathrm{P}_{r e p U}-n e o:: m y c\right]$

B. subtilis BBG125 Mycosubtilin monoproducer BBG116 derivative; This work 


$$
\begin{aligned}
& \mathrm{Myc}+\text { Srf- Amy } \operatorname{Spc}^{\mathrm{R}} \mathrm{Nm}^{\mathrm{R}} \mathrm{Cm}^{\mathrm{R}} \mathrm{Tc}^{\mathrm{R}} ; \\
& {\left[\mathrm{P}_{\text {repU-口eo: }: \text { myc; srfAA::cat-tet }]}\right.}
\end{aligned}
$$

Fungi
Botrytis cinerea
Wild-type
Lab stock
Fusarium oxysporum
Wild-type
Lab stock

Yeast

Saccharomyces cerevisiae Wild-type

Lab stock

Plasmids

pGEM-T Easy

T-vector; $\mathrm{Ap}^{\mathrm{R}} ; 3.0 \mathrm{~kb}$

Promega Corp.,

Madison, WI, USA

ED511

Bacillus integration vector with the

Rudner, D.Z.,

Pspac-comK-spc cassette; $\mathrm{Spc}^{\mathrm{R}} ; 7.8 \mathrm{~kb}$

Boston, MA, USA

pBC16

Staphylococcus/Bacillus cloning vector;

Bernhard et al.

$\mathrm{Tc}^{\mathrm{R}} ; 4.6 \mathrm{~kb}$

pC194

Staphylococcus/Bacillus cloning vector;

Ehrlich (1977)

$\mathrm{Cm}^{\mathrm{R}} ; 2.9 \mathrm{~kb}$

pTnMod-RKm'

Plasposon; $\mathrm{Km}^{\mathrm{R}} / \mathrm{Nm}^{\mathrm{R}} ; 4.8 \mathrm{~kb}$

Dennis and Zylstra

(1998)

pUC19

Cloning vector, $\mathrm{Ap}^{\mathrm{R}} ; 2.7 \mathrm{~kb}$

New England

Biolabs, Beverly,

MA, USA

pBG106

pUC19 containing PrepU-neo inserted

Leclère et al. (2005) 
within PCR-amplified flanking regions of

$m y c$ promoter; $\mathrm{Ap}^{\mathrm{R}} \mathrm{Nm}^{\mathrm{R}} ; 5.3 \mathrm{~kb}$

pBG201

NspI-SacI fragment from pTnMod-RKm'

This work

ligated to the $S p h \mathrm{I}-S a c \mathrm{I}\left[\mathrm{P}_{r e p U}-n e o\right]$ one

from $\mathrm{pBG} 106 ; \mathrm{Nm}^{\mathrm{R}} ; 3.1 \mathrm{~kb}$

pBG208

EsrfAA amplicon inserted within pGEM-T

This work

Easy; $\mathrm{Ap}^{\mathrm{R}} ; 5.2 \mathrm{~kb}$

pBG210

EsrfAA amplicon subcloned within pUC19; This work

$\mathrm{Ap}^{\mathrm{R}} ; 4.9 \mathrm{~kb}$

pBG209

tet gene amplicon from $\mathrm{pBC} 16$ inserted

This work

within pGEM-T Easy; $\mathrm{Ap}^{\mathrm{R}} \mathrm{Tc}^{\mathrm{R}} ; 4.6 \mathrm{~kb}$

pBG212

pUC19 derivative containing part of srfAA

This work

gene from ATCC 6633 interrupted by the

tet gene of $\mathrm{pBC} 16 ; \mathrm{Ap}^{\mathrm{R}} \mathrm{Tc}^{\mathrm{R}} ; 6.5 \mathrm{~kb}$

pBG143

cat gene amplicon from $\mathrm{pC} 194$ inserted

This work

within pGEM-T Easy; $\mathrm{Ap}^{\mathrm{R}} \mathrm{Cm}^{\mathrm{R}} ; 3.7 \mathrm{~kb}$

pBG144

cat gene from $\mathrm{pC} 194$ inserted within the

This work

end of tet gene in $\mathrm{pBG} 212 ; \mathrm{Ap}^{\mathrm{R}} \mathrm{Cm}^{\mathrm{R}} \mathrm{Tc}^{\mathrm{R}}$

$7.2 \mathrm{~kb}$

${ }^{*} \mathrm{Ap}^{\mathrm{R}} ; \mathrm{Cm}^{\mathrm{R}} ; \mathrm{Nm}^{\mathrm{R}} ; \mathrm{Spc}^{\mathrm{R}} ; \mathrm{Tc}^{\mathrm{R}}$; resistances to ampicillin, chloramphenicol, neomycin,

spectinomycin and tetracycline, respectively. Srf- Pps-: unable to synthesize both surfactin and plipastatin. Amy- : unable to degrade starch (amylase negative). 


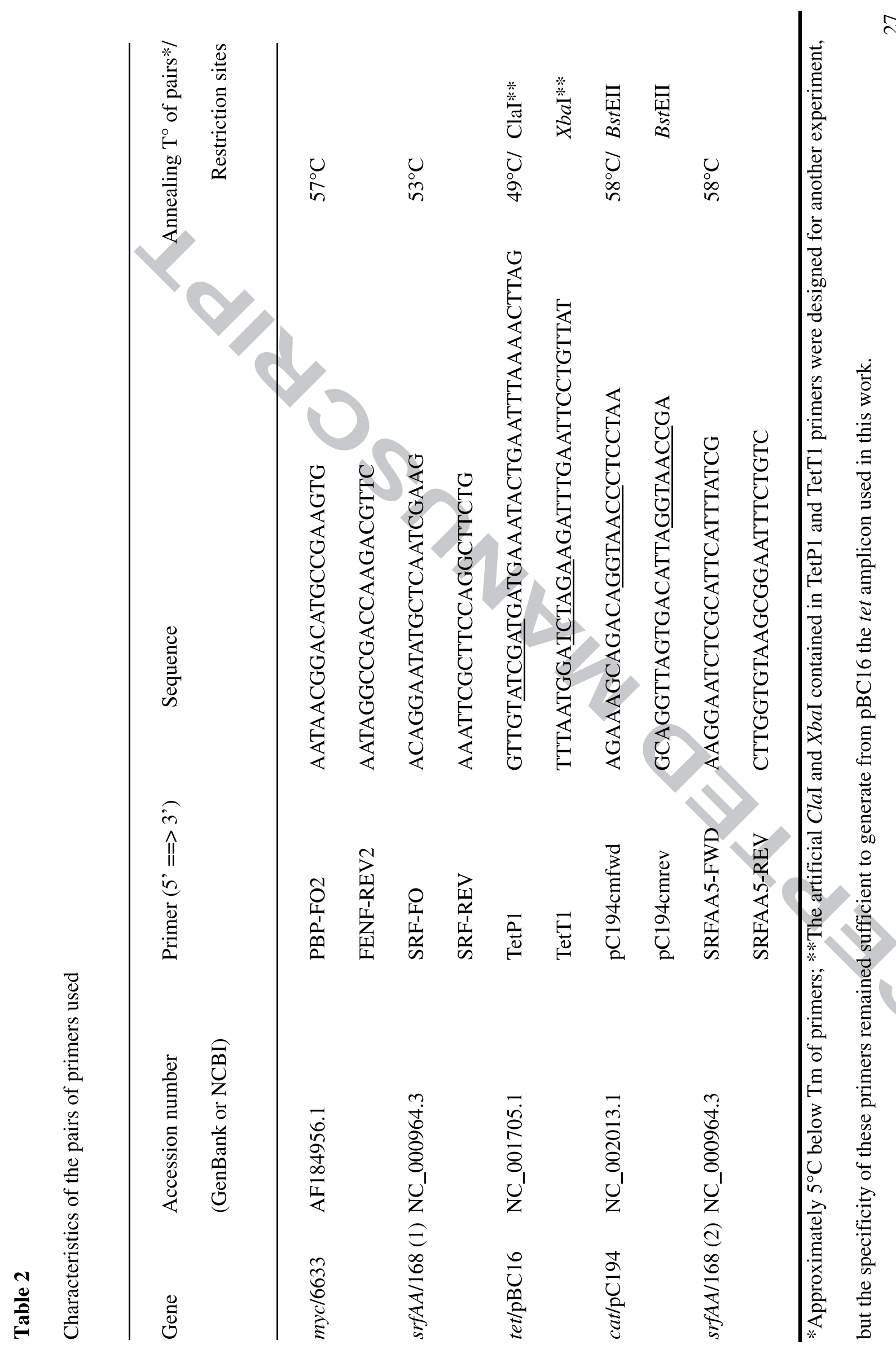


Table 3.

Growth rates in the early and late stages of exponential phase, mycosubtilin maximal production and specific productivity of BBG125 at different growth temperatures.*

\begin{tabular}{lllll}
\hline $\mathrm{T}^{\circ}$ & \multicolumn{2}{c}{ Growth rate } & Maximal & Maximal specific \\
& Early stage & Late stage & production & productivity \\
& & & $(\mathrm{mg} / \mathrm{L})^{\alpha}$ & $(\mathrm{ng} / \text { cell })^{\alpha}$ \\
& & & & \\
\hline $30^{\circ} \mathrm{C}$ & $0.40 \pm 4.4 \mathrm{E}-02$ & $0.07 \pm 6.9 \mathrm{E}-03$ & $52.70 \pm 3.53$ & $33.15 \pm 1.48$ \\
$22^{\circ} \mathrm{C}$ & $0.17 \pm 2.2 \mathrm{E}-03$ & $0.02 \pm 1.9 \mathrm{E}-03$ & $104.83 \pm 9.57$ & $132.25 \pm 3.83$ \\
$15^{\circ} \mathrm{C}$ & $0.04 \pm 0.1 \mathrm{E}-03$ & $0.01 \pm 0.6 \mathrm{E}-03$ & $58.44 \pm 7.47$ & $103.02 \pm 5.9$ \\
\hline
\end{tabular}

*The values are means from three independent experiments. ${ }^{\text {x }}$ These values were obtained $48 \mathrm{~h}$ after the beginning of the stationary phase.

\section{Table 4.}

$\mathrm{m} / \mathrm{z}$ (mass-to-charge ratio) of the 10 peaks detected by combined HPLC and MALDI/TOF analyses and correspondence with mycosubtilin forms.

\begin{tabular}{llllll}
\hline Peak no. & $\mathrm{m} / \mathrm{z}$ & Form & Peak no. & $\mathrm{m} / \mathrm{z}$ & Form \\
1 & 1056 & $n-\mathrm{C} 15$ & 6 & 1098 & anteiso-C17 Gln \\
2 & 1084 & iso-C16 Gln & 7 & 1098 & iso-C17 Gln \\
3 & 1084 & $n$-C16 Gln & 8 & 1084 & anteiso-C17 \\
4 & 1070 & iso-C16 & 9 & 1084 & iso-C17 \\
5 & 1070 & $n$-C16 & 10 & 1098 & $n$-C18 \\
\hline
\end{tabular}


Table 5.

Increasing $\mathrm{m} / \mathrm{z}$ (mass-to-charge ratio) obtained with the fragmentation $\mathrm{m} / \mathrm{z} 1260.4$ ion.

\begin{tabular}{llllll}
\hline $\mathbf{m} / \mathbf{z}$ & Fragment ion & $\mathbf{m} / \mathbf{z}$ & Fragment ion & $\mathbf{m} / \mathbf{z}$ & Fragment ion
\end{tabular}

382.3: $\mathrm{xN}$

566.5: PSNx

791.5: $\mathrm{b} 6-\mathrm{NH}_{3}$

462.3: $\mathrm{NxN}-2 \mathrm{NH}_{3}$

645.3: $\mathrm{PSNxN}-\mathrm{H}_{2} \mathrm{O}-\mathrm{NH}_{3} \quad$ 808.4: $\mathrm{b} 6$

479.3: $\mathrm{a} 5-2 \mathrm{NH}_{3}$

646.6: $\mathrm{PSNxN}-2 \mathrm{NH}_{3}$

831.1: y4

489.2: $\mathrm{b} 5-\mathrm{H}_{2} \mathrm{O}-2 \mathrm{NH}_{3}$

663.5: $\mathrm{PSNxN}-\mathrm{NH}_{3}$

871.5: b7-3NH

506.2: b5- $\mathrm{H}_{2} \mathrm{O}-\mathrm{NH}_{3}$

680.5: PSNXN

888.5: $b 7-2 \mathrm{NH}_{3}$

524.2: b5 $-\mathrm{NH}_{3}$

757.3: b6- $3 \mathrm{NH}_{3}$

905.5: $\mathrm{b} 7-\mathrm{NH}_{3}$

541.2: b5

774.5: b6- $2 \mathrm{NH}_{3}$

1015.2: y6 

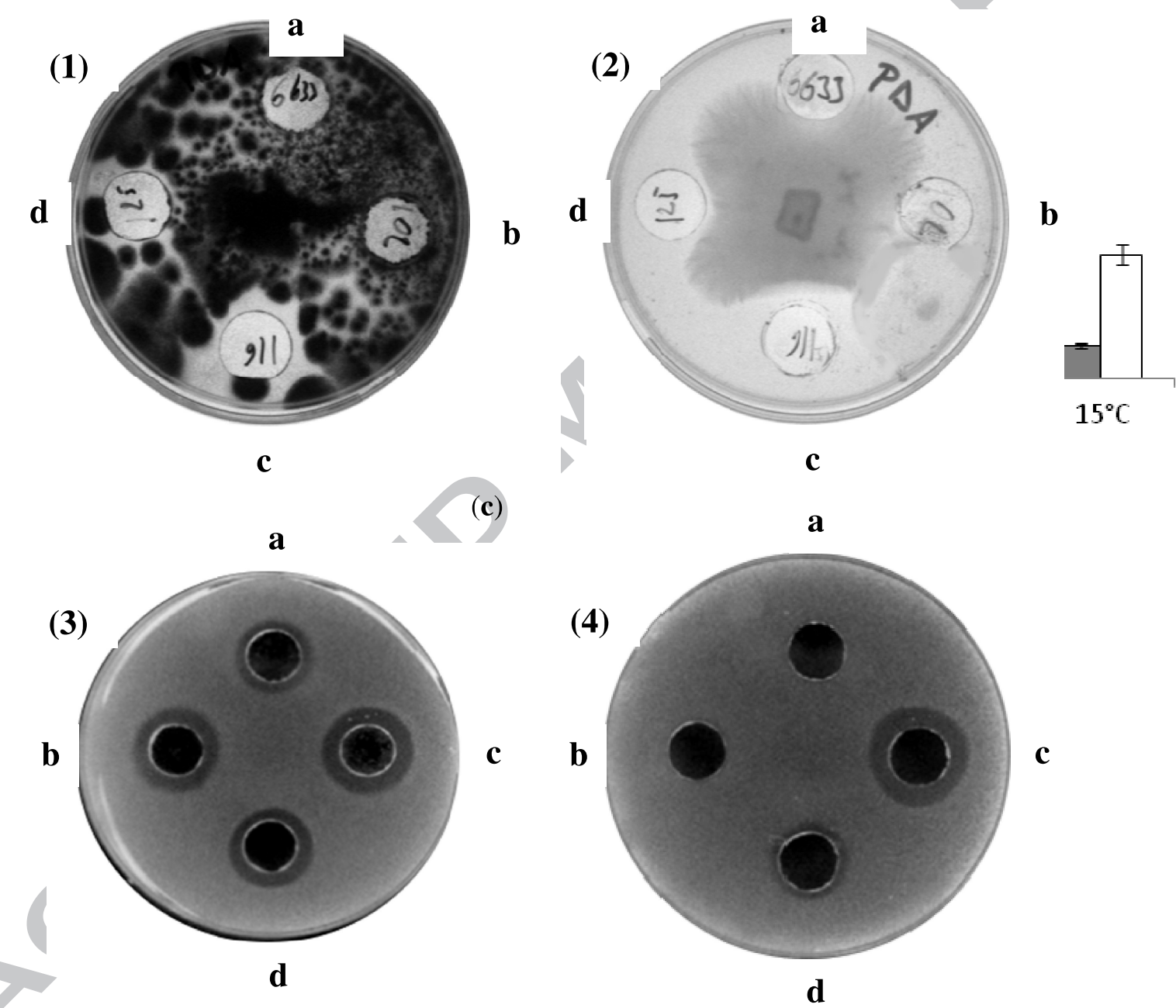


\section{FIGURE}

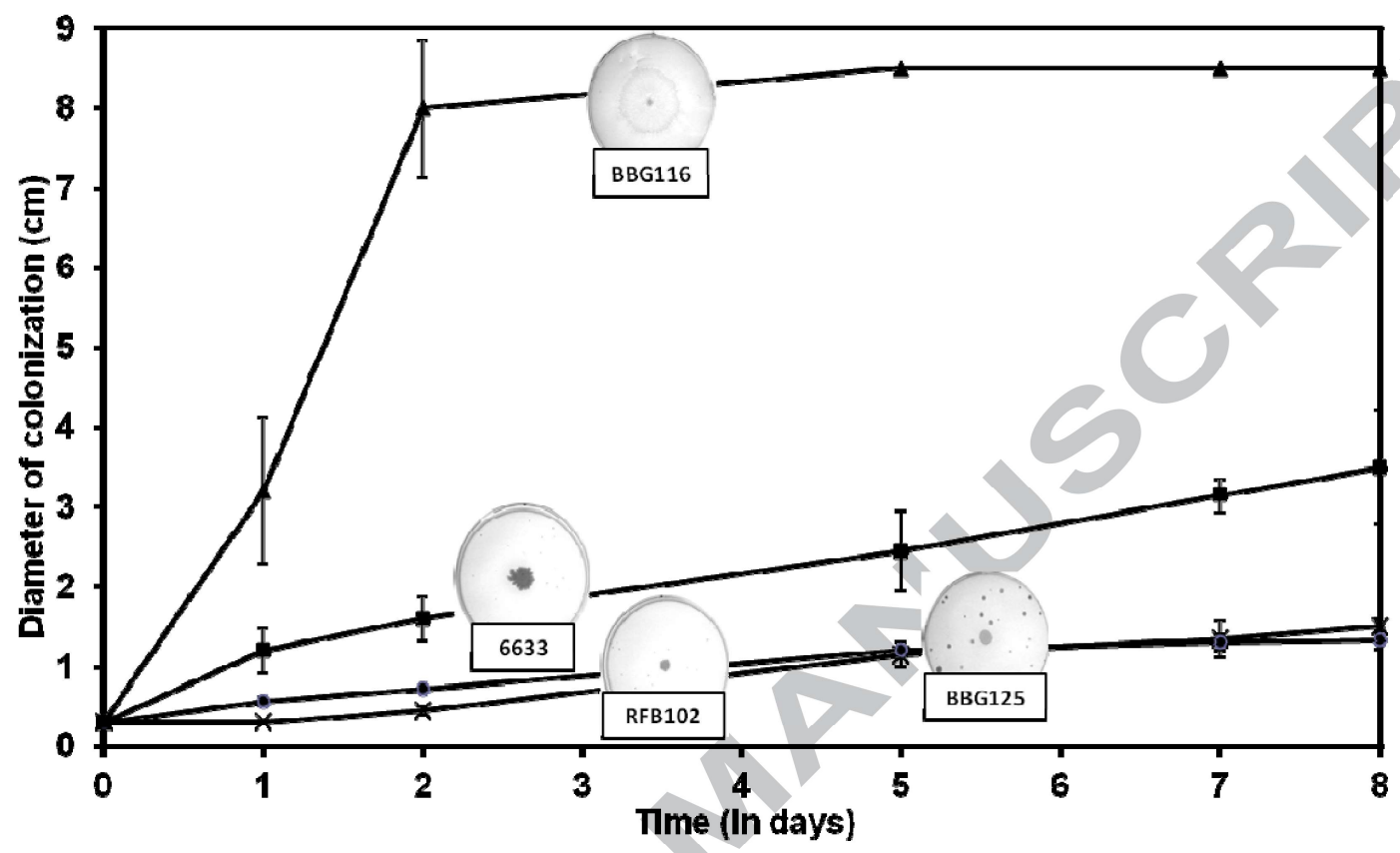

Fig. 1 


\section{LEGEND OF FIGURE}

Fig. 1. (B) Comparison of the invasive behaviour of B. subtilis ATCC 6633 and its derivatives on soft B medium. ATCC 6633; — RFB102; $\boldsymbol{\Delta}$ BBG116; × BBG125. 


\section{Highlights}

- mutant strain which constitutively produces mycosubtilin only

- new mixture of mycosubtilins containing five new isoforms

- influence of surfactin gene knock-out on mycosubtilin production

- influence of strain modification on fatty acid and mycosubtilin isoform pattern 